\section{What do we really know about UK paediatric dental general anaesthesia services?}

\author{
S. Robertson, ${ }^{1}$ A. Ni Chaollai ${ }^{2}$ and T. A. Dyer ${ }^{3}$
}

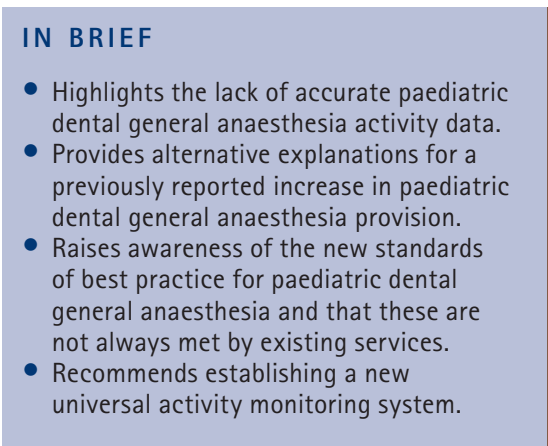

Dental general anaesthesia (DGA) is only permitted within a hospital setting where critical care facilities are available. Recently, concern has been expressed about the number of hospital admissions for the dental care of children following the publication of a high profile paper which highlighted an apparent increase in children being admitted for extractions due to caries under DGA. Coincidentally new best practice standards for paediatric DGA services have been published. An evaluation of DGA services in Yorkshire and the Humber suggested that existing monitoring was inadequate and is unlikely to represent true levels of activity and that any apparent increase may reflect the method of remuneration for services. In fact, recent changes in service structure and changes to improve quality have reduced DGA activity in some areas. In addition, the evaluation revealed that many services were not meeting standards of best practice.

\section{INTRODUCTION AND BACKGROUND}

Historically DGA was provided in general dental practice, by primary salaried dental services (formerly the community dental service) and in hospital settings. However, it has undergone considerable change in response to safety concerns following DGA-related deaths, many of which occurred in general dental practice. Recommendations from regulatory bodies initiated changes which led to DGA being restricted to hospital settings from 31 December 2001. ${ }^{1-4}$ Recent guidelines which broadly complement each other have proposed further standards for services to meet., ${ }^{5,6}$

Before DGA ceased in general dental practice, activity was recorded by the Dental Practice Board (DPB). Episodes of care in the community dental services

"Specialist Registrar in Dental Public Health, NHS Sheffield, 722 Prince of Wales Road, Sheffield, S9 4EU; ${ }^{2}$ Paediatric Dentist in Private Practice, The Northbrook Clinic, 15A Northbrook Road, Ranelagh, Dublin 6, Ireland; ${ }^{3}$ Honourary Lecturer in Dental Public Health, School of Clinical Dentistry, University of Sheffield, 19 Claremont Crescent Sheffield, S10 2TA

*Correspondence to: Dr Sarah Robertson Email:s.robertson7@nhs.net

\section{Refereed Paper}

Accepted 5 January 2011

DOI: 10.1038/sj.bdj.2012.138

${ }^{\circledR}$ British Dental Journal 2012; 212: 165-167 which involved a general anaesthetic (GA) or sedation were also recorded until 1999. ${ }^{1}$ This enabled assessment and analysis of variations and trends in DGA provision in primary care across the country. ${ }^{7-10}$ In contrast, DGA activity in hospitals has been unclear. The Hospital Episode Statistics (HES) database was established in 1987, ${ }^{11}$ and DGA activity has been estimated by analysing numbers of ordinary admissions and day cases seen by dental specialities, the majority of which are likely to have involved treatments under GA. ${ }^{1}$

A recent high profile study reported an increasing trend in hospital admissions for dental care in children in England between 1997 and 2006, linked to a 66\% increase in extractions for caries. ${ }^{12}$ The authors analysed activity data recorded on the HES database for patients up to 17 years old, whose primary diagnosis was a dental condition. They assumed that most of the episodes of care (particularly extractions) were performed under DGA.

\section{INACCURACY IN HES DATA}

In light of this apparent increase in DGA provision and the publication of best practice standards, an evaluation of DGA services was undertaken in Yorkshire and the Humber. It aimed to establish the location, organisation and monitoring systems of paediatric DGA services, and used a postal survey of all DGA service providers. The main findings were that there was no universal data monitoring system, and that there was much variation in the way services were being run, which often did not meet standards of best practice. ${ }^{13}$

The HES system was used to record DGA activity in just over half (56\%) of DGA lists, about a fifth (21.3\%) were unaware of the method of data recording, and the remainder listed ten 'other' methods. As almost half of the lists did not use the HES system, this suggests that much activity was not captured.

An analysis of HES data was also performed in parallel with the survey, with the aim of investigating the relative provision of DGA in different parts of Yorkshire and the Humber. ${ }^{14}$ Data were extracted by the Yorkshire and Humber Public Health Observatory from the National HES database. ${ }^{11}$ The number of inpatient and day case admissions for dental procedures provided was determined for children under 16 years of age between 1 April 2007 and 31 March 2008 for each provider. The number of admissions was used as a proxy for the number of DGA procedures provided, given that for this age group most dental procedures carried out in hospitals 
are performed under DGA. All possible HES codes associated with extractions, restorative treatment and minor oral surgery were included in the search criteria. Each incidence of one of these codes was recorded as being associated with a DGA experience. If a child underwent more than one of these procedures during a single stay in hospital, this was still recorded as a single DGA experience. However, if one of these codes was recorded for a subsequent hospital stay, then each was counted as an additional DGA experience. For each it was also determined whether a separate HES code for the use of GA had been applied. A similar search was undertaken for the same codes for outpatient attendances, as some GAs may be provided on an outpatient basis such that a patient may go straight into theatre from a waiting area and then spend a short time in the recovery room before being discharged.

HES data for inpatient and day case DGA in Yorkshire and the Humber suggested that around 2,764 were performed by 22 providers, with only one provider separately specifying that a GA had been involved for a small number of its admissions. The outpatient data were poorly coded and could only be described in terms of type of attendance. One provider recorded 53 first attendances which could involve a GA, however, none of the other providers recorded more than five.

There was a discrepancy between DGA activity recorded using the HES system (around 2,817) and that estimated by the postal questionnaire $(13,500) .{ }^{13}$ As approximately $44 \%$ of lists did not record activity on the HES database, it is likely that this was the main cause of the discrepancy. Interestingly even different lists provided within the same hospital used a variety of recording mechanisms. Furthermore, many providers who did record HES data reported much lower levels of activity than that estimated by the survey, and some HES data were recorded for hospitals where paediatric DGA was not provided.

Although Moles and Ashley ${ }^{12}$ acknowledged possible inaccuracies in HES data recording, they may not have been aware that activity was not always recorded using the HES system. Their data are likely to underestimate DGA provision. They reported a rising trend in admissions

Table 1 Proportion of DGA lists not meeting standards of best practice ${ }^{13}$

\begin{tabular}{|c|c|}
\hline Standard of best practice & Proportion not meeting standard \\
\hline Referral protocol & $34.7 \%$ \\
\hline $\begin{array}{l}\text { Pre-operative treatment planning assessment on separate day to } \\
\text { the DGA }\end{array}$ & $22.7 \%$ \\
\hline $\begin{array}{l}\text { Comprehensive care (all necessary treatment performed under a } \\
\text { single GA) }\end{array}$ & $28.0 \%$ \\
\hline Arrangements for ongoing preventive care following discharge & $53.3 \%$ \\
\hline
\end{tabular}

which was consistent with an earlier study, ${ }^{15}$ and postulated that this may be linked to two factors. Firstly, activity may have increased since all DGA now has to be administered in secondary care following the publication of $A$ conscious decision. ${ }^{1}$ Secondly, a reduction in restorative care for children in primary care may have resulted in increased referrals.

However, our research suggests this trend could be explained by an increase in the numbers of services using the HES system to record activity over the same time period. This may be a consequence of the HES system being used by hospitals to claim payment for activity through 'payment by results. ${ }^{16}$ In addition, the process of Transforming Community Services resulted in many DGA lists previously run by salaried dental services within a hospital setting being subsumed into hospital services. The activity from these lists would not have been captured in the past but has started to appear in HES data.

Moles and Ashley ${ }^{12}$ reported that most procedures undertaken were not separately recorded as having involved a GA. As this concurs with our findings, we share their concern. An additional worry is that some treatments are coded inaccurately. For example, surgical extractions of wisdom teeth were sometimes recorded for children, which may reflect the lack of knowledge of data recorders who are often non-clinical staff. As HES data are commonly used as part of the method of remuneration for provision there could also be an incentive to allocate codes for more highly paid procedures.

New standards for DGA services have been proposed by recent DGA guidelines. ${ }^{5,6}$ It could be argued that DGA activity may be decreasing as the guidelines emphasise the need to try all other avenues before opting for DGA. The introduction of referral protocols, pre-operative dental assessments for treatment planning, comprehensive care and a focus on prevention should also reduce the numbers of children being treated under DGA and repeat GAs.

The Royal College of Surgeons' guidelines encourage a holistic comprehensive care approach to DGA, such that the child is rendered dentally fit by the end of a single GA. ${ }^{5}$ Any restorative or other dental care required in addition to the extractions should take place either under local anaesthetic before or at the same time as extractions under DGA. This move towards comprehensive care restricts the numbers of children that can be seen on lists and hence the amount of DGA being provided. Where standards of best practice have been adopted, providers also report anecdotal reductions in DGA provision.

Furthermore many DGA services were lost in the process of Transforming Community Services ${ }^{17}$ and there is uncertainty over the longevity of others. Our evaluation highlighted that many services will need to be reconfigured if they are to meet standards of best practice (Table 1), ,6, 13 and some may no longer be viable. This may compound the existing inequalities in waiting times and availability of restorative care under DGA. ${ }^{13,17}$

\section{A NATIONAL PICTURE OF DGA}

Given the interest in improving standards of paediatric DGA, the costs to the NHS and the personal costs to children and parents/carers who experience DGA, it is concerning that activity levels are unknown. We propose that a universal activity monitoring system should be established that is not linked to any system of remuneration. The data would provide a new baseline from which to monitor levels of activity in different areas and analyse trends.

One aim of the new general dental services contract pilots is to improve 
child dental care from a preventive and treatment perspective. Monitoring DGA activity will be an important part of any evaluation of the impact of the contract on patient care. It would also inform the development and evaluation of community oral health promotion schemes such as water fluoridation.

Furthermore, there are no national data on the location of, access to and quality of services, and treatment pathways. Our study suggested that in Yorkshire and the Humber referrals may be related to decay experience within populations, availability and access to DGA services and whether there are paediatric services providing alternatives such as inhalation sedation. ${ }^{14}$ The availability of NHS primary dental care and the willingness and competence of practitioners to treat children is also likely to have an impact. We propose that a national overview of DGA services would provide a good basis to monitor the quality of existing services, highlight potential training needs and guide equitable planning of new services.

\section{RECOMMENDATIONS}

In order to gain an overview of UK paediatric DGA services, we recommend that a universal monitoring system for DGA is introduced, and that the Department of Health considers commissioning a national audit and service evaluation of DGA services, looking at access to services, quality, provision and need.

The authors would like to thank the Yorkshire and Humber Public Health Observatory for providing HES data.

1. Department of Health. A conscious decision: a review of the use of general anaesthesia and conscious sedation in primary dental care. London: Department of Health, 2000.

2. Clinical Standards Advisory Group. Dental general anaesthesia. London: HMSO, 1995.

3. General Dental Council. Maintaining standards. Guidance to dentists on professional and personal conduct London: GDC 1998.

4. General Dental Council. Standards for dental professionals. London: GDC, 2005.

5. Davies $C$. Harrison M Roberts G. UK national clinical guidelines in paediatric dentistry: guideline for the use of general anaesthesia (GA) in paediatric dentistry London: Royal College of Surgeons of England, 2008. Online article available at $h t t p: / / w w w . r c s e n g . a c . u k / f d s / p u b l i c a t i o n s-$ clinical-guidelines/clinical_guidelines/documents/ Guideline\%20for\%20the\%20use \%20of\%20 GA\%20in\%20Paediatric\%20Dentistry\%20May\%20 2008\%20Final.pdf (accessed September 2011).

6. Adewale L, Morton N, Blayney M. Guidelines for the management of children referred for dental extractions under general anaesthesia. London: Association of Paediatric Anaesthetists of Great Britain and Ireland, 2011. Online article available at http://www.apagbi.org.uk/sites/apagbi.org.uk/ files/Main\%20Dental\%20Guidelines\%5B1\%5D.pdf (accessed September 2011).

7. The Dental Observatory. Dental general anaesthesia and sedation in the north of England 1991/92 to 2001/02. Preston: The Dental Observatory, 2003.
8. Whittle J G, Woods K. Dental general anaesthesia in the north of England 1991/1992 to 1999/2000. The Dental Public Health Northern Quality Improvement Group, 2001

9. Whittle J G. The provision of primary care dental general anaesthesia and sedation in the north west region of England 1996-1999. Br Dent J 2000; 189: 500-502.

10. Murray J J. General anaesthesia and children's dental health: present trends and future needs. Anaesth Pain Control Dent 1993; 2: 209-216.

11. The NHS Information Centre for Health and Social Care. Hospital Episode Statistics (HES). HESonline, 2011. Online information available at http://www.hesonline.nhs.uk/Ease/servlet/Conten tServer?sitelD=1937\&tcategorylD=537 (accessed October 2011).

12. Moles D R, Ashley P. Hospital admissions for dental care in children: England 1997-2006. Br Dent J 2009; 206: E14.

13. Ni Chaollai A, Robertson S, Dyer T A, Balmer R C Fayle S A. An evaluation of paediatric dental general anaesthesia in Yorkshire and the Humber. Br Dent J 2010; 209: E20.

14. Robertson S. Audit and service evaluation of paediatric dental general anaesthetic services in Yorkshire and the Humber. Sheffield: University of Sheffield, 2008. MDPH dissertation.

15. Thomas $S$ J, Atkinson $C$, Hughes $C$, Revington $P$, Ness A R. Is there an epidemic of admissions for surgical treatment of dental abscesses in the UK? BMJ 2008; 336: 1219-1220.

16. Department of Health. Payment by results. Online information available at http://www.dh.gov.uk/ health/category/policy-areas/nhs/resources-formanagers/payment-by-results/ (accessed January 2012).

17. British Society of Paediatric Dentistry. Report of a BSPD members' survey in response to 'Transforming Community Services.' BSPD, 2011. Online article available at http://www.bspd.co.uk/ LinkClick.aspx?fileticket=ALrXQqLOxOM\%3d\&tta bid $=40$ (accessed October 2011) 